\title{
MicroRNAs in Barrett's esophagus: future prospects
}

\author{
Juntaro Matsuzaki ${ }^{1,2}$ and Hidekazu Suzuki ${ }^{2 *}$ \\ ${ }^{1}$ Center for Preventive Medicine, Keio University Hospital, Tokyo, Japan \\ ${ }^{2}$ Division of Gastroenterology and Hepatology, Department of Internal Medicine, Keio University School of Medicine, Tokyo, Japan \\ *Correspondence: hsuzuki@a6.keio.jp \\ Edited by: \\ Yoshimasa Saito, Keio University Faculty of Pharmacy, Japan \\ Reviewed by: \\ Jun Yasuda, The JFCR-Cancer Institute, Japan
}

Keywords: Barrett's esophagus, esophageal adenocarcinoma, microRNA, biomarkers, prediction

Esophageal adenocarcinoma is an aggressive malignancy with a poor prognosis. In Western countries, the incidence of esophageal adenocarcinoma has increased dramatically in the last three decades. To improve patient survival and reduce disease burden, early-stage detection, or better yet, preventing the progression of esophageal adenocarcinoma from its premalignant lesions, constitute the best short-term options. Barrett's esophagus is histologically characterized by the replacement of the normal stratified squamous epithelium of the esophagus with a columnar epithelium with intestinal differentiation (Matsuzaki et al., 2010, 2011). Barrett's esophagus is considered to be a complication of gastroesophageal reflux disease and a precursor lesion of esophageal adenocarcinoma. It is generally believed that the progression of Barrett's esophagus involves a series of histological changes: non-dysplastic Barrett's metaplasia, low-grade dysplasia, high-grade dysplasia, and ultimately, adenocarcinoma. Although these features justify endoscopic surveillance for the premalignant stages, patients with Barrett's esophagus show an absolute annual risk of only $0.12 \%$ for the development of esophageal adenocarcinoma (Hvid-Jensen et al., 2011). Therefore, recommending the invasive and expensive conventional endoscopic screening procedure is deemed controversial. In fact, Corley et al. reported that, within a large community-based population, endoscopic surveillance of Barrett's esophagus was not associated with a substantial decrease in the risk of death from esophageal adenocarcinoma (Corley et al., 2013). Thus, identification of better risk stratification biomarkers to determine the risk of progression from Barrett's esophagus to esophageal adenocarcinoma may improve disease outcome and make patient management more cost-efficient.

MicroRNAs (miRNAs) are a class of small non-coding endogenous RNAs, 18-25 nucleotides in length, and are capable of simultaneous regulation of genes by binding to target mRNAs, resulting in mRNA degradation or translational inhibition. miRNAs participate in many essential biological processes, including proliferation, differentiation, apoptosis, necrosis, autophagy, and stress responses (Saito et al., 2011b, 2012a). miRNAs have also been shown to play a potential role in cancer pathogenesis through their functions as oncogenes or tumor suppressors, depending on their gene targets (Saito et al., 2009a, 2011a; Nishizawa and Suzuki, 2013). Compared to mRNAs, miRNAs are less numerous in humans and have been proposed to act as better biomarkers by virtue of their small size, greater stability, and capability of regulating hundreds of mRNAs. Therefore, miRNAs profiling could improve the risk stratification for the progression of Barrett's esophagus to esophageal adenocarcinoma.

MiRNAs can be profiled on a genomewide scale using array or sequencing technologies. However, very few studies have been conducted to identify miRNAs as prognostic biomarkers for the progression of Barrett's esophagus to adenocarcinoma. Although several cross-sectional studies using comprehensive array analysis have been reported (Feber et al., 2008; Kan et al., 2009; Yang et al., 2009; Fassan et al., 2011; Leidner et al., 2012; Wu et al., 2013), their results have proved controversial. They compared the expression of
miRNAs across different types of histological specimens such as Barrett's esophagus, low-grade dysplasia, high-grade dysplasia, and esophageal adenocarcinoma, and reported that a substantial number of miRNAs show differential expression in esophageal tissues (Sakai et al., 2013). Indeed, they might be useful in revealing certain mechanisms underlying carcinogenesis. But, they might be difficult to identify risk stratification biomarkers. We should think about much better research strategies.

Recently, two nice studies were reported to identify risk stratification biomarkers for Barrett's esophagus: one prospective study and one cross-sectional study. First, Revilla-Nuin et al. have reported a set of miRNAs associated with this progression and provided further validation in two groups of patients with Barrett's esophagus, who either developed or did not develop adenocarcinoma, over a course of 5 years (Revilla-Nuin et al., 2013). Among 24 patients with Barrett's esophagus, 7 patients progressed to adenocarcinoma while the other 17 did not. Four miRNAs (miR-192, miR-194, miR-196a, and miR196b) were found to show significantly higher expression in patients with progression to esophageal adenocarcinoma than in patients who did not show disease progression. Second, Saad et al. conducted a notable comprehensive microarray profiling for identifying the specific miRNA signature associated with esophageal adenocarcinoma (Saad et al., 2013). They analyzed 13 samples from isolated Barrett's esophagus, 10 from Barrett's esophagus adjacent to high-grade dysplasia, 17 from high-grade dysplasia, and 34 from esophageal adenocarcinoma tissue. They 
identified that $m i R-21, m i R-31, m i R-192$, and miR-194 were upregulated in Barrett's esophagus adjacent to high-grade dysplasia lesions as compared to isolated Barrett' esophagus. In addition, these 4 miRNAs were upregulated in a progressive manner through the Barrett's metaplasia-dysplasia-adenocarcinoma sequence. More importantly, this study provided findings for Barrett's esophagus for two groups: isolated Barrett's esophagus vs. Barrett's esophagus adjacent to high-grade dysplasia. The limitations of both two papers include the very small sample size. Larger prospective multiinstitutional studies are warranted to confirm this result. Another criticism against the studies using comprehensive microarray analysis is that these could not provide the insights how miRNAs may exert their effects (Saito et al., 2009b, 2012b, 2013).

Since clinical predictors of increased risk of esophageal adenocarcinoma, namely, the length of Barrett's esophagus, male gender, older age, current tobacco smoking, alcohol consumption, central obesity, and bile reflux, have been established, the association between the expression levels of miRNA in Barrett's esophagus and these clinical risk factors would require further investigation. We had recently reported that expression levels of $m i R-221$ and miR-222 increased when cultured esophageal epithelial cells were exposed to bile acids. miR-221 and $m i R-222$ are known to specifically target p27Kip1, which in turn inhibits the proteasomal protein degradation of CDX2 (caudal-related homolog 2) (Matsuzaki et al., 2013). Furthermore, $m i R-221$ and $m i R-222$ expressions are higher in esophageal adenocarcinoma than in the surrounding Barrett's esophagus. We also confirmed that the levels of p27Kip1 and CDX2 were lower in areas of esophageal adenocarcinoma than in those of Barrett's esophagus. Thus, we showed that the degradation of CDX2 was enhanced by upregulation of miR-221 and miR-222 on exposure to bile acids. Although bile acids are known to induce DNA damage, resistance to apoptosis through NF- $\mathrm{KB}$ activation, and resistance to autophagy (Fang et al., 2013), the association between bile acids and miRNA expression has never been reported except for our results (Masaoka and Suzuki, 2014). In this way, clinical epidemiological information would be important and useful to reveal novel insights of miRNA in the progression of Barrett's esophagus to adenocarcinoma.

In conclusion, on the basis of clinical importance, better risk stratification biomarkers to determine the risk of progression from Barrett's esophagus to esophageal adenocarcinoma are expected. We should deepen our knowledge of miRNA using clinical materials, hopefully with more prospective approach. The fusion of basic science and clinical science research would also be required for identifying the upstream regulation and the downstream targets of miRNAs and understanding their mode of action. These will facilitate the development of miRNAbased prevention or therapeutic strategies for esophageal adenocarcinoma.

\section{ACKNOWLEDGMENTS}

This work was supported by a Grant-inAid for Scientific Research (B) from the Japan Society for the Promotion of Science (JSPS) (22300169, to Hidekazu Suzuki), a grant from the Smoking Research Foundation (to Hidekazu Suzuki), the Research Fund of Daiwa Securities Health Foundation (to Hidekazu Suzuki), and a Grant-in-Aid for JSPS Fellows (to Juntaro Matsuzaki).

\section{REFERENCES}

Corley, D. A., Mehtani, K., Quesenberry, C., Zhao, W., De Boer, J., and Weiss, N. S. (2013). Impact of endoscopic surveillance on mortality from Barrett's esophagus-associated esophageal adenocarcinomas. Gastroenterology 145, 312-319.e311. doi: 10.1053/j.gastro.2013.05.004

Fang, Y., Chen, X., Bajpai, M., Verma, A., Das, K. M., Souza, R. F., et al. (2013). Cellular origins and molecular mechanisms of Barrett's esophagus and esophageal adenocarcinoma. Ann. N.Y. Acad. Sci. 1300, 187-199. doi: 10.1111/nyas.12249

Fassan, M., Volinia, S., Palatini, J., Pizzi, M., Baffa, R., De Bernard, M., et al. (2011). MicroRNA expression profiling in human Barrett's carcinogenesis. Int. J. Cancer 129, 1661-1670. doi: 10.1002/ijc. 25823

Feber, A., Xi, L., Luketich, J. D., Pennathur, A., Landreneau, R. J., Wu, M., et al. (2008). MicroRNA expression profiles of esophageal cancer. J. Thorac. Cardiovasc. Surg. 135, 255-260. discussion: 260. doi: 10.1016/j.jtcvs.2007.08.055

Hvid-Jensen, F., Pedersen, L., Drewes, A. M., Sorensen, H. T., and Funch-Jensen, P. (2011). Incidence of adenocarcinoma among patients with Barrett's esophagus. N. Engl. J. Med. 365, 1375-1383. doi: 10.1056/NEJMoa1103042
Kan, T., Sato, F., Ito, T., Matsumura, N., David, S., Cheng, Y., et al. (2009). The miR-106b-25 polycistron, activated by genomic amplification, functions as an oncogene by suppressing p21 and Bim. Gastroenterology 136, 1689-1700. doi: 10.1053/j.gastro.2009.02.002

Leidner, R. S., Ravi, L., Leahy, P., Chen, Y., Bednarchik, B., Streppel, M., et al. (2012). The microRNAs, MiR-31 and MiR-375, as candidate markers in Barrett's esophageal carcinogenesis. Gene. Chromosome. Canc. 51, 473-479. doi: 10.1002/gcc.21934

Masaoka, T., and Suzuki, H. (2014). Does bile reflux influence the progression of Barrett's esophagus to adenocarcinoma? (Gastroenterology 2013;145:1300-1311). J. Neurogastroenterol. Motil. 20, 124-126. doi: 10.5056/jnm.2014.20.1.124

Matsuzaki, J., Suzuki, H., Asakura, K., Saito, Y., Hirata, K., Takebayashi, T., et al. (2010). Gallstones increase the prevalence of Barrett's esophagus. J. Gastroenterol. 45, 171-178. doi: 10.1007/s00535009-0153-4

Matsuzaki, J., Suzuki, H., Asakura, K., Saito, Y., Hirata, K., Takebayashi, T., et al. (2011). Etiological difference between ultrashort- and short-segment Barrett's esophagus. J. Gastroenterol. 46, 332-338. doi: 10.1007/s00535-010-0353-y

Matsuzaki, J., Suzuki, H., Tsugawa, H., Watanabe, M., Hossain, S., Arai, E., et al. (2013). Bile acids increase levels of microRNAs 221 and 222, leading to degradation of CDX2 during esophageal carcinogenesis. Gastroenterology 145, 1300-1311. doi: 10.1053/j.gastro.2013.08.008

Nishizawa, T., and Suzuki, H. (2013). The Role of microRNA in gastric malignancy. Int. J. Mol. Sci. 14, 9487-9496. doi: 10.3390/ijms14059487

Revilla-Nuin, B., Parrilla, P., Lozano, J. J., De Haro, L. F., Ortiz, A., Martinez, C., et al. (2013). Predictive value of MicroRNAs in the progression of barrett esophagus to adenocarcinoma in a long-term follow-up study. Ann. Surg. 257, 886-893. doi: 10.1097/SLA.0b013e31826ddba6

Saad, R., Chen, Z., Zhu, S., Jia, P., Zhao, Z., Washington, M. K., et al. (2013). Deciphering the unique microRNA signature in human esophageal adenocarcinoma. PLoS ONE 8:e64463. doi: 10.1371/journal.pone.0064463

Saito, Y., Suzuki, H., and Hibi, T. (2009a). The role of microRNAs in gastrointestinal cancers. J. Gastroenterol. 44(Suppl. 19), 18-22. doi: 10.1007/s00535-008-2285-3

Saito, Y., Suzuki, H., Tsugawa, H., Nakagawa, I., Matsuzaki, J., Kanai, Y., et al. (2009b) Chromatin remodeling at Alu repeats by epigenetic treatment activates silenced microRNA-512$5 \mathrm{p}$ with downregulation of $\mathrm{Mcl}-1$ in human gastric cancer cells. Oncogene 28, 2738-2744. doi: 10.1038/onc. 2009.140

Saito, Y., Suzuki, H., Imaeda, H., Matsuzaki, J., Hirata, K., Tsugawa, H., et al. (2013). The tumor suppressor microRNA-29c is downregulated and restored by celecoxib in human gastric cancer cells. Int. J. Cancer 132, 1751-1760. doi: 10.1002/ijc.27862

Saito, Y., Suzuki, H., Matsuura, M., Sato, A., Kasai, Y., Yamada, K., et al. (2011a). MicroRNAs in hepatobiliary and pancreatic cancers. Front. Genet. 2:66. doi: 10.3389/fgene.2011.00066

Saito, Y., Suzuki, H., Tsugawa, H., Suzuki, S., Matsuzaki, J., Hirata, K., et al. (2011b). 
Dysfunctional gastric emptying with downregulation of muscle-specific microRNAs in Helicobacter pylori-infected mice. Gastroenterology 140, 189-198. doi: 10.1053/ j.gastro.2010.08.044

Saito, Y., Suzuki, H., Taya, T., Nishizawa, M., Tsugawa, H., Matsuzaki, J., et al. (2012a). Development of a novel microRNA promoter microarray for ChIPon-chip assay to identify epigenetically regulated microRNAs. Biochem. Biophys. Res. Commun. 426, 33-37. doi: 10.1016/j.bbrc.2012.08.012

Saito, Y., Suzuki, H., Tsugawa, H., Imaeda, H., Matsuzaki, J., Hirata, K., et al. (2012b). Overexpression of miR-142-5p and miR-155 in Gastric Mucosa-Associated Lymphoid Tissue (MALT) lymphoma resistant to helicobacter pylori eradication. PLoS ONE 7:e47396. doi: 10.1371/journal.pone.0047396

Sakai, N. S., Samia-Aly, E., Barbera, M., and Fitzgerald, R. C. (2013). A review of the current understanding and clinical utility of miRNAs in esophageal cancer. Semin. Cancer Biol. 23, 512-521. doi: 10.1016/j.semcancer.2013. 08.005

Wu, X., Ajani, J. A., Gu, J., Chang, D. W., Tan, W. Hildebrandt, M. A., et al. (2013). MicroRNA expression signatures during malignant progression from Barrett's esophagus to esophageal adenocarcinoma. Cancer Prev. Res. (Phila) 6, 196-205. doi: 10.1158/1940-6207. CAPR-12-0276

Yang, H., Gu, J., Wang, K. K., Zhang, W., Xing, J., Chen, Z., et al. (2009). MicroRNA expression signatures in Barrett's esophagus and esophageal adenocarcinoma. Clin. Cancer Res. 15, 5744-5752. doi: 10.1158/1078-0432.CCR09-0385

Conflict of Interest Statement: The authors declare that the research was conducted in the absence of any commercial or financial relationships that could be construed as a potential conflict of interest.

Received: 04 February 2014; accepted: 17 March 2014; published online: 03 April 2014.

Citation: Matsuzaki J and Suzuki H (2014) MicroRNAs in Barrett's esophagus: future prospects. Front. Genet. 5:69. doi: 10.3389/fgene.2014.00069

This article was submitted to Epigenomics and Epigenetics, a section of the journal Frontiers in Genetics.

Copyright () 2014 Matsuzaki and Suzuki. This is an open-access article distributed under the terms of the Creative Commons Attribution License (CC BY). The use, distribution or reproduction in other forums is permitted, provided the original author(s) or licensor are credited and that the original publication in this journal is cited, in accordance with accepted academic practice. No use, distribution or reproduction is permitted which does not comply with these terms. 